\title{
Analysis of OFDM WDM using Direct Detection Technique
}

\author{
Vyankatesh Maske ${ }^{1}$, Pawase Ramesh $\mathrm{S}^{1}$ and R.P.Labade ${ }^{1}$ \\ ${ }^{1}$ Electronics and Telecommunication Department \\ Amrutvahini college of Engineering, Sangamner, India \\ vyanky24@gmail.com
}

\begin{abstract}
This paper presents Long Reach Orthogonal frequency Division multiplexing Wavelength Division Multiplexing (OFDM-WDM) system capable of delivering downstream $40 \mathrm{Gbit/s}$ data. The optical source for downstream data is $\mathrm{CW}$ Laser at central office. We use four OFDM transmitter at central office for the 40-Gb/s downstream transmission. We have used direct detection technique for OFDM and did analysis of OFDM WDM using direct detection technique for without compensation and with compensation. We extend the maximum reach of this WDM PON to be $96 \mathrm{~km}$ by using Erbium-doped fiber amplifiers at the RN. Bit error rate was measured and constellation diagram observed to demonstrate the proposed scheme. In this paper Long reach and large data service aspects of a OFDM WDM is presented. The results show that the error-free transmission can be achieved for all WDM channels with sufficient power margins.
\end{abstract}

Keywords: Wavelength division multiplexing (WDM), orthogonal frequency division multiplexing (OFDM), Erbium doped fiber amplifier (EDFA), Single mode fiber (SMF), Photo detector $(P D)$

\section{Introduction}

In order to deliver future broadband services economically, service providers have to reduce the cost in order to sustain profit margins. One possible way to achieve this is to simplify the network architecture; hence the number of equipment interfaces and network elements, e.g. electronic switches and routers, can be reduced. Extended reach (ER) access network has been proposed to achieve this [1-3]. The ER access network makes use of a high capacity, high split-ratio passive optical network (PON), with a reach of more than $100 \mathrm{~km}$, to combine optical access and metro into a single system [3]. The local exchange [between the head-end office and the customer optical networking units (ONUs)] would contain a small amount of compact, low power physical layer elements, such as optical amplifiers and temperature controlled arrayed waveguide gratings for wavelength multiplexing and demultiplexing. The higher layer networking equipments would be located in a small number of head-end offices (also called centralized major service nodes). Gigabit PONs (GPONs) are now standardized and commercially available. They typically provide a split-ratio of 32 via passive optical splitters using time division multiplexing (TDM), over a reach of up to $20 \mathrm{~km}$. They offer 1 to $2.5 \mathrm{~Gb} / \mathrm{s}$ downstream and $\sim 1 \mathrm{~Gb} / \mathrm{s}$ upstream data rate. The proposed ER-PON usually requires four new features: (1) high data rate in both upstream and downstream directions $(>1 \mathrm{~Gb} / \mathrm{s}$ ); (2) reach extension to $\sim 100 \mathrm{~km}$; (3) a high split ratio (> 100); and (4) using wavelength division multiplexing (WDM). It is viable to directly use $10 \mathrm{~Gb} / \mathrm{s}$ system in these ERPONs. This would require the adoption of relatively expensive $10 \mathrm{GHz}$ optical components (if on-off-keying modulation is used), such as optical modulator or semiconductor optical amplifier (SOA), in the cost sensitivity ONU. The cost of the ONU is not shared as it is, for example, the cost of optical line terminal (OLT). In this work, we 
propose and demonstrate WDM ER-PONs that makes use of orthogonal frequency division multiplexing (OFDM). Due to the highly spectral efficiency of the M-quadrature amplitude modulation (QAM) in each subcarrier of the OFDM signal, low-bandwidth optical components can still be used. This means we can directly increase the data rate of the system while using the existing optical components developed for GPONs. Besides, the inherent advantage of OFDM frequency diversity transmission allows simple equalization of frequency response by baseband digital signal processing (DSP) [4]. These characteristics can be used to mitigate fiber chromatic dispersion $[5,6]$ which is one of the major impairments in ER-PONs).

In this work, we propose and demonstrate a long-reach orthogonal frequency division multiplexing (OFDM) wavelength-division-multiplexed (WDM) PON capable of providing $40-\mathrm{Gb} / \mathrm{s}$ service to each subscriber using direct detection technique. Thus, the $40-\mathrm{Gb} / \mathrm{s}$ downstream signal is obtained by combining the outputs of four OFDM transmitters at central office using the WDM technique.

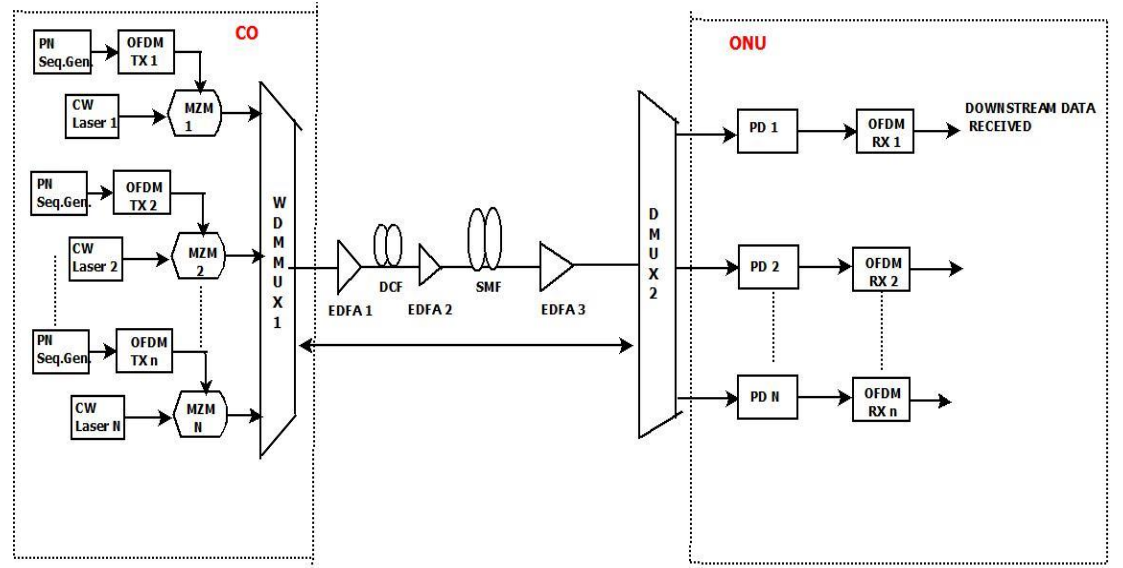

Figure 1. The Schematic Diagram of the Proposed DD-OFDM WDM

\section{Operational Principle}

Figure 1 shows principle of the proposed OFDM WDM. We are going to use Continuous wave $(\mathrm{CW})$ laser as light source

Which generate light of difference wavelengths. At the central office, continuous wave (CW) optical signals at difference wavelength with specified peak power level are generated. In this paper, external modulation technique is used to improve the performance of communication system. The PN sequence generator generates a downstream data which consist a stream of bit sequence, At the transmitter, this downstream data is given to OFDM transmitter where the OFDM transmitter produces the electrical OFDM signal which is up converted into the optical domain by the electrical to optical (E/O) up

Converter which does intensity modulation. The downstream data is externally modulated over continuous wave optical signal using Mach- Zehnder modulator as external modulator .Downstream data which is generated using OFDM transmitter are transmitted over continuous wave optical signals and given to wavelength division multiplexing 1 (WDM 1) which is used for multiplexing of downstream signal of difference wavelengths. All these downstream signals are transmitted via EDFA 1, dispersion compensating fiber (DCF), EDFA 2, single mode fiber (SMF) and EDFA 3 .As shown in fig. 1 EDFA 3 and DMUX 1 are used in Remote node. Downstream signals are transmitted via EDFA 1 to strengthen weaken signals, to make it available for long distance and then after these are transmitted via DMUX 1 which is used for a purpose of demultiplexing of downstream signal and are transmitted to Optical Network Unit (ONU). 
At the receiver, the incoming optical signal is converted to the electrical domain by an optical to electrical $(\mathrm{O} / \mathrm{E})$ converter, which is in this case a photodiode. The received electrical signal is demodulated using the OFDM demodulator with similar parameters as the OFDM modulator, the guard interval is removed. After that the signal is fed into a mQAM decoder, and the BER is calculated at the end.

\section{Experimental Set - Up}

This paper demonstrates a downstream data 40-Gb/s OFDM WDM with the experimental setup shown in Figure 2. For downstream transmission, PN sequence generator generates 10 Gbps. Here, we have designed OFDM WDM system [7-9] for 4 users. This generated bit sequence which is in series applied to serial to parallel converter. The parallel converted bites applied to m-QAM encoder. In this project we are taking 2 bits per symbol and generating a symbol of two bits per symbol such QAM encoder is 4QAM. The bit stream is generated by a PRBS generator and mapped by a 4-QAM encoder These generated symbols are applied to OFDM Modulator which is inverse Fourier transform IFFT OFDM .The resulting signal is modulated by an OFDM modulator which having parameter number of subcarrier equal to 8, cylix prefix equal to 0.25 .CW Lasers which are operating at wavelength $\lambda 1$ equal $1549 \mathrm{~nm}, \lambda 2$ equal to 1550 $\mathrm{nm}, \lambda 3$ equal to $1551 \mathrm{~nm}$ and $\lambda 4$ equal to $1552 \mathrm{~nm}$ with input optical power equal to OdBm. CW Lasers have line width equal to $10 \mathrm{MHz}$.
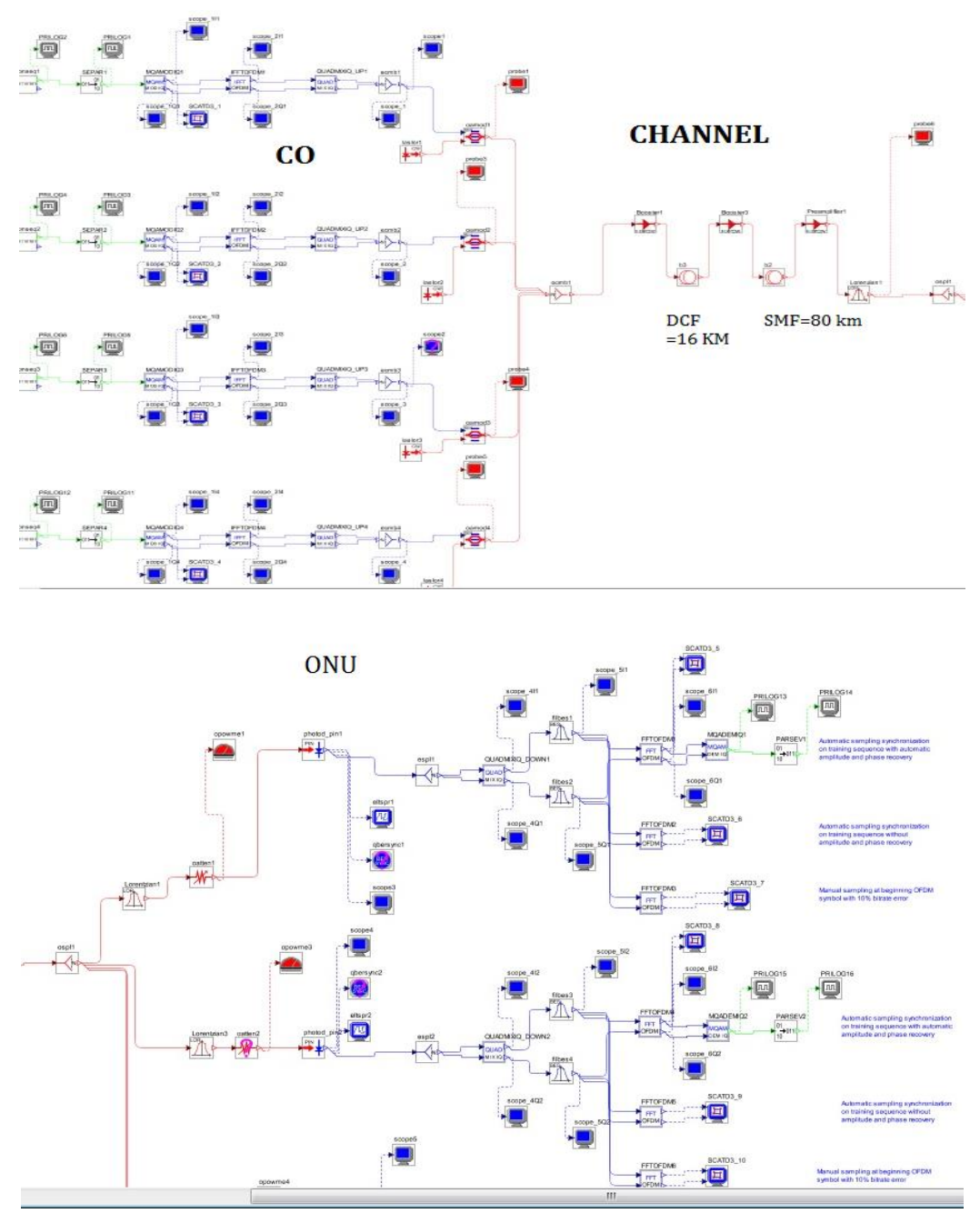

Figure 2. Experimental Set-Up for Proposed Scheme DD-OFDM 
After that, the resulting electrical signal is modulated to the optical signal using a Mach-Zehnder modulators (MZM). The resulting optical signals after the four MZMs which will be multiplexed using WDM MUX 1. After multiplexing; those entire four signals (40Gbps $=10 \mathrm{Gbps} * 4$ ) are transmitted via EDFA 1, EDFA 2, EDFA 3. The attenuation of SMF is balanced by optical amplifier which is EDFA. These EDFAs are having gain equal to $35 \mathrm{~dB}$ and noise figure equal to $10 \mathrm{~dB}$. A single mode optical fiber of length $80 \mathrm{~km}$ is used .There are three EDFAs are used to strengthen weak downstream signal. The dispersion parameters for $\mathrm{SMF}$ at $1550 \mathrm{~nm}$ are $16 \mathrm{ps} / \mathrm{nm} / \mathrm{km}$ and $0.05938 \mathrm{ps} / \mathrm{nm}^{2} / \mathrm{km}$. The loss in SMF is $0.2 \mathrm{~dB} / \mathrm{km}$. Here DCF is used in order to reduce the dispersion in SMF. The dispersion parameters for DCF at $1550 \mathrm{~nm}$ are $-80 \mathrm{ps} / \mathrm{nm} / \mathrm{km}$ and $0.16743 \mathrm{ps} / \mathrm{nm}^{2} / \mathrm{km}$. The loss in DCF is $0.6 \mathrm{~dB} / \mathrm{km}$. The length of DCF is $16 \mathrm{~km}$. Further, all those four difference wavelengths signal are demultiplexed by DEMUX 1 .The demultiplexed signals, at the receiver(ONU) the incoming optical signal is converted to the electrical domain by an optical to electrical $(\mathrm{O} / \mathrm{E})$ converter, which is in this case a PIN photodiode. The parameters of PIN photodiode are quantum efficiency equal to 0.7 , responsivity equal to $0.8751 \mathrm{~A} / \mathrm{W}$ and dark current equal to $0.1 \mathrm{nA}$. After down converting the signal, it passes through a low-pass filter (LPF) and is transmitted to the OFDM receiver to get the original signal. The output of low pass filter is given to FFT OFDM.FFT OFDM work in opposite to IFFT OFDM, remove the guard band. The output of FFT OFDM is given to 4-QAM decoder .The output of decoder is given to parallel to serial converter and finally we can receive the transmitted data.

\section{Results and Discussions}

We have implemented OFDM WDM using direct detection technique as shown in figure 2.We have considered 4 user WDM system. We implemented the system using optsim version 13 software and considered three cases 1.without dispersion compensation 2.with pre compensation technique 3 .with post compensation technique $.80 \mathrm{~km}$ length SMF used for without compensation technique. It is observed that the performance of OFDM WDM for given case is worst .The implemented system is failure if we consider the first case of without compensation .We have shown the Constellation diagram of 4QAM for without compensation technique in fig.4 .After that, We have considered the post compensation technique for given OFDM WDM system .We have observed the constellation diagram for 4 wavelength signal which is shown in fig 5.If we compare the constellation diagram for without compensation and with post compensation technique, it is observed that constellation diagram for with post compensation technique is good one The constellation diagram is without distortion. We have calculated the bit error rate (BER) for 4 wavelengths signal. We have changed the input optical power from $0 \mathrm{dBm},-5$ $\mathrm{dBm},-10 \mathrm{dBm},-15 \mathrm{dBm}$ and measured the received optical power for 4 wavelengths signals and measured the respective BER for that received optical power .We observed the BER in the range of $1 \mathrm{E}-06$ to $1 \mathrm{E}-08$ which is good one. The measured value of input optical power, received optical power for respective wavelength, BER for respective wavelength is shown in Table 2. After post compensation technique we have implemented OFDM WDM system using pre compensation technique and tried to observe constellation diagram for 4 wavelengths signal .The constellation diagram for 4 wavelength signal of 4QAM for with pre compensation technique is shown in figure 6.If we compare the constellation diagram for three cases without compensation, with post compensation and with pre compensation technique, we observed constellation diagram for pre compensation technique is good one compare to others two. We have calculated the bit error rate (BER) for 4 wavelengths signal .We have changed the input optical power from $0 \mathrm{dBm},-5 \mathrm{dBm},-10 \mathrm{dBm},-15 \mathrm{dBm}$ and measured the received optical power for respective 4 wavelengths signals and measured the respective BER for that received optical power. We observed the BER in the range of $1 \mathrm{E}-09$ to $1 \mathrm{E}-13$ which is good one.The measured value 
of input optical power, received optical power for respective wavelength, BER for respective wavelength is shown in Table 1. The measured BER for pre compensation is good compare to post compensation. Finally it can be said OFDM WDM system with pre compensation gave good performance in terms of BER, constellation diagram. We have compared between a OFDM WDM system with 4-QAM and 16-QAM.In Figure 6, we have shown Constellation diagram of 4-QAM for with pre compensation technique for 4 wavelength signals and in Figure 7 ,a constellation diagram for 16-QAM system is shown for 4 wavelength signals. It is observed that constellation diagram for 4-QAM system is good and with less dispersion but constellation diagram for 16-QAM is with more dispersion which give more BER.

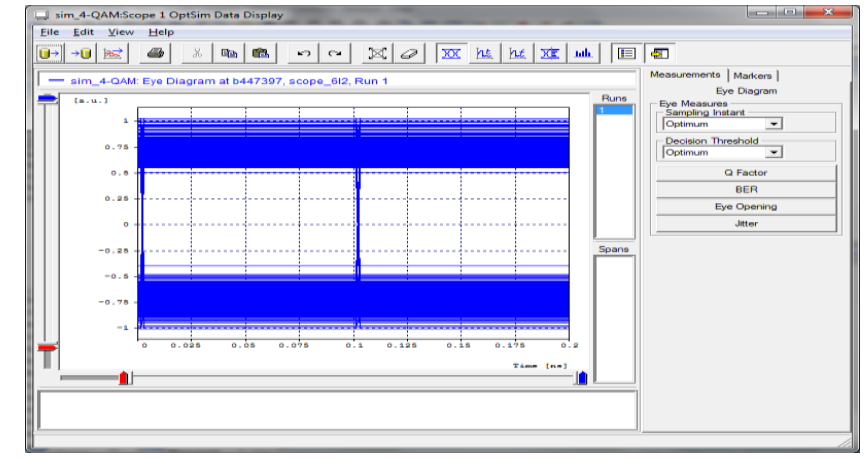

Figure 3. Eye Diagram for $1550 \mathrm{Nm}$ Channel for Pre Compensation Technique

Table 1. Downstream Data Performance Analysis with Pre Dispersion Compensation

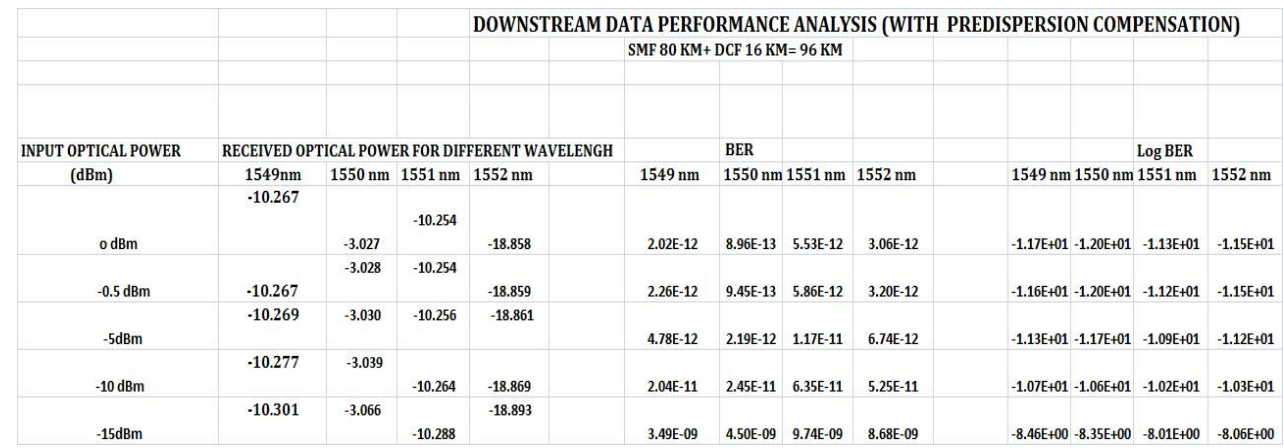

Table 2. Downstream Data Performance Analysis with Post Dispersion Compensation

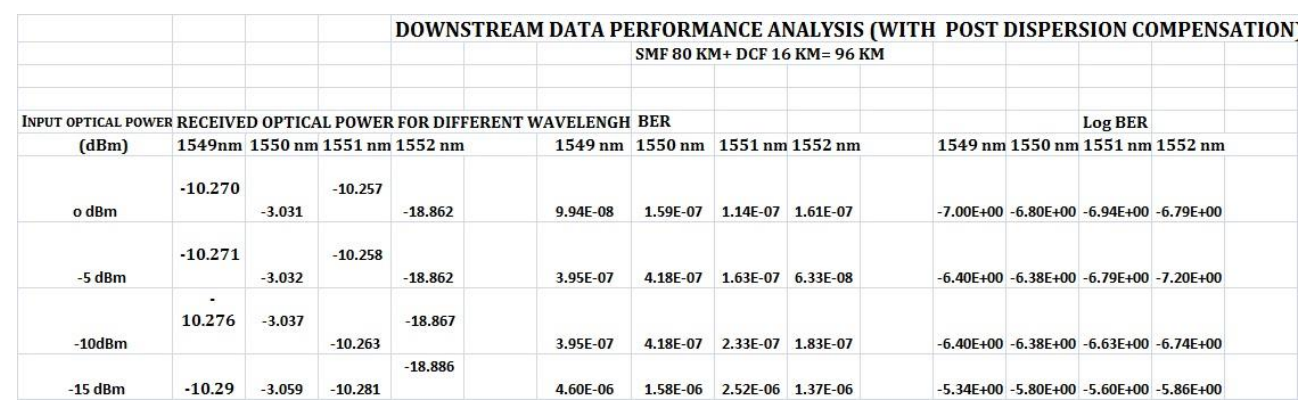




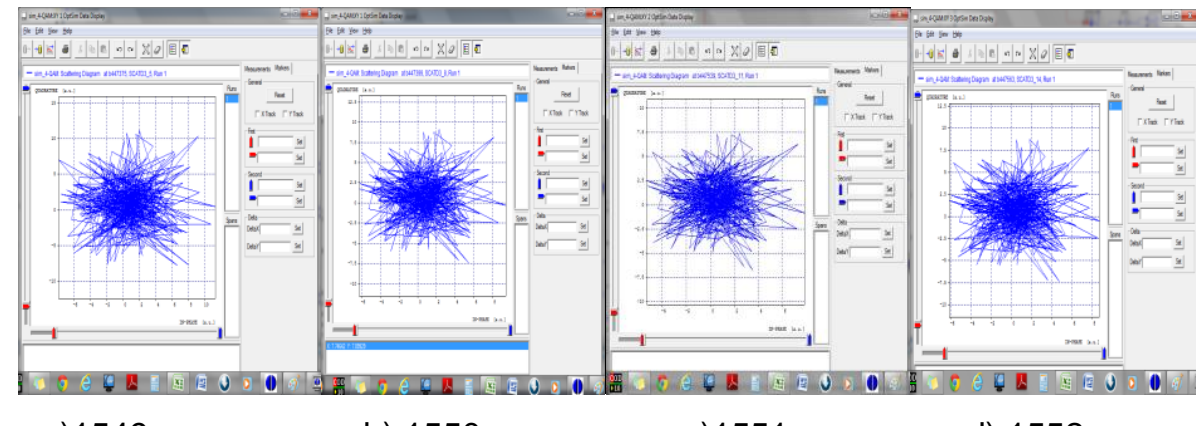
a) $1549 \mathrm{~nm}$
b) $1550 \mathrm{~nm}$
c) $1551 \mathrm{~nm}$
d) $1552 \mathrm{~nm}$

Figure 4. Constellation Diagram of 4-QAM for without Compensation Technique

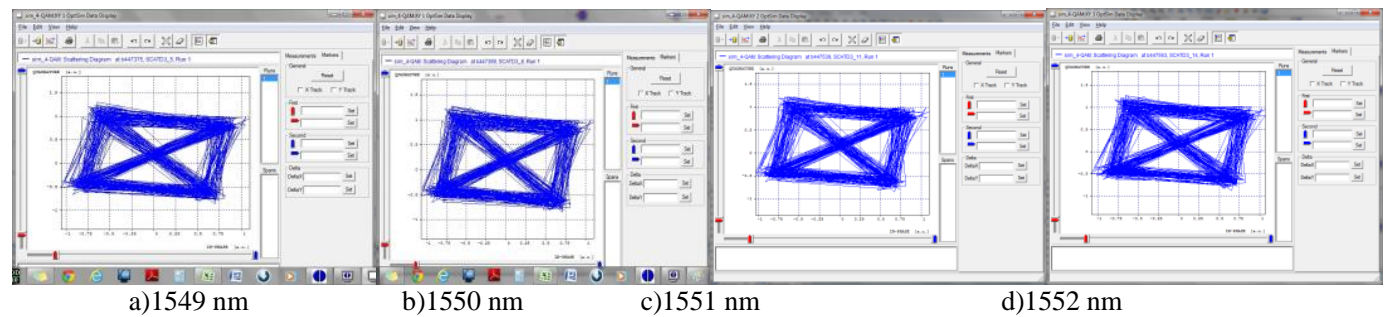

Figure 5. Constellation Diagram of 4-QAM for with Post Compensation Technique

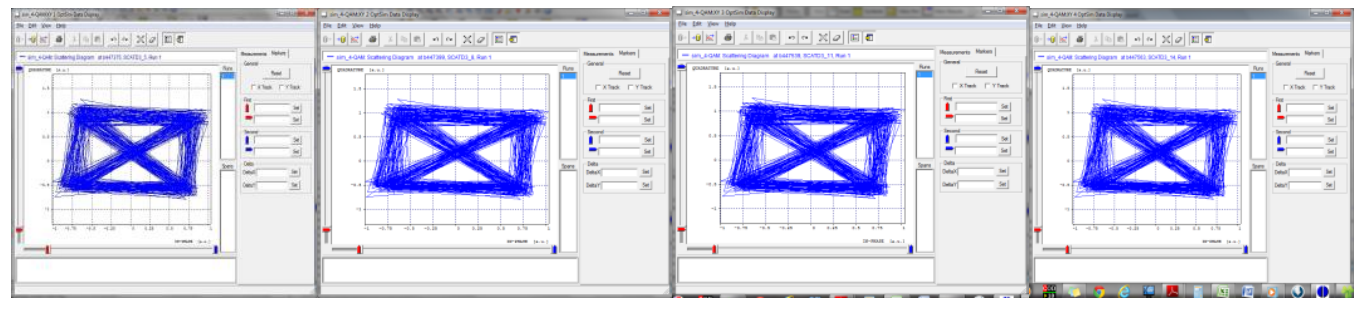
a) $1549 \mathrm{~nm}$
b) $1550 \mathrm{~nm}$
c) $1551 \mathrm{~nm}$
d) $1552 \mathrm{~nm}$

Figure 6. Constellation Diagram of 4-QAM for with Pre Compensation Technique

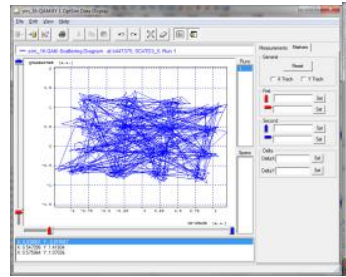

a) $1549 \mathrm{~nm}$

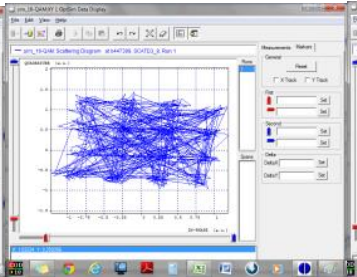

b) $1550 \mathrm{~nm}$

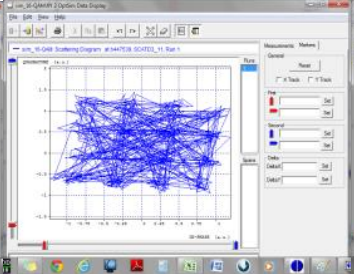

c) $1551 \mathrm{~nm}$

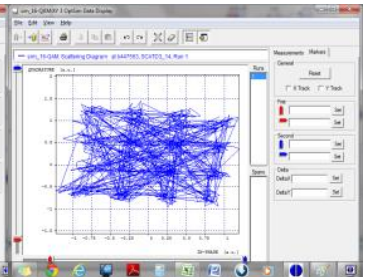

d) $1552 \mathrm{~nm}$

Figure 7. Constellation Diagram Of 16-QAM For With Pre Compensation Technique

It is successfully demonstrated that Long Reach Orthogonal frequency wavelength division multiplexing (OFDM WDM) system can be successfully implemented for 96 $\mathrm{Km}$. It delivers downstream 40-Gbps data. We have implemented OFDM WDM using without compensation and with compensation technique. We have experimentally demonstrated that a OFDM WDM system with direct detection technique can give good performance for pre compensation technique. The results show that the error-free transmission can be achieved for all WDM channels with sufficient power margins. 


\section{References}

[1] D. P. Shea, A.D. Ellis, D.B. Payne, R.P. Davey and J. E. Mitchell, "10 Gbit/s PON with 100 km reach and x1024 split," Proc. of ECOC, Rimini, Italy, Paper We, (2003), p. 147.

[2] G. Talli and P.D. Townsend, "Hybrid DWDM-TDM long-reach PON for next-generation optical access", J. Lightwave, Technol., vol. 24, (2006), pp. 2827-2834.

[3] P.D. Townsend, G. Talli, C.W. Chow, E.M. MacHale, C. Antony, R. Davey, T. De Ridder, X. Z. Qiu, P. Ossieur, H. G. Krimmel, D. W. Smith, I. Lealman, A. Poustie, S. Randel and H. Rohde, "Long Reach Passive Optical Networks" IEEE LEOS Annual Meeting, Invited Paper, Florida, USA, (2007).

[4] Y.M. Lin, "Demonstration and design of high spectral efficiency 4Gb/s OFDM system in passive optical networks", Proc. of OFC, Paper OThD7, (2007).

[5] J.M. Tang and K. Alan, "30-Gb/s signal transmission over 40-km directly modulated DFB-laser-based single-mode-fiber links without optical amplification and dispersion compensation", J. Lightwave. Technol., vol. 24, (2006), pp. 2318-2326.

[6] N.E. Jolley, H. kee, R. Rickard, J. Tang and K. Cordina, "Generation and propagation of a 1550nm $10 \mathrm{Gbit} / \mathrm{s}$ optical orthogonal frequency division multiplexed signal over $1000 \mathrm{~m}$ of multimode fiber using a directly modulated DFB", Proc. of OFC, Paper OFP3, (2005).

[7] L. Tawade, "100 Gb/s Long- Reach WDM-PON Implemented By Using Directly Modulated RSOA", Microwave Optical Technology Letter, vol. 55, no.6, (2013), pp. 1426-1430.

[8] L. Tawade, S. Mhatli and R. Attia, "Bidirectional long reach WDM-PON delivering downstream data 20Gbps and upstream data 10Gbps using mode locked laser and RSOA”, Optical Quantum Electron DOI 10.1007/s11082-014-9952-9.

[9] L. Tawade, S. Mhatli, M. Ghanbarisabagh and R. Attia, "Long-reach OFDM WDM-PON delivering $100 \mathrm{~Gb} / \mathrm{s}$ of data downstream and $2 \mathrm{~Gb} / \mathrm{s}$ of data upstream using a continuous-wave laser and a reflective semiconductor optical amplifier", Optics Letters, vol. 39, no. 23, (2014). 
International Journal of Future Generation Communication and Networking Vol. 8, No. 6 (2015) 